\title{
New design approach to MIMO nonlinear controller for direct expansion air conditioning system in building automation system
}

\begin{abstract}
This paper introduce a new approach for implementation of soft computing methodology of fuzzy cognitive map to control the parameters of Direct Expansion (DX) air-conditioning (A/C) systems. In the past few decades, the application of different controllers on heating, ventilating and air-conditioning system and also air conditioning system separately are considered due to a high demand of these appliances in the buildings and high energy consumption in the building automation system. Designing the suitable fuzzy cognitive map (FCM) controller on air conditioning system could increase more energy efficiency and also more energy saving in building automation system. The simple FCM control algorithm by using generalized predictive control (GPC) for assigning the weights could obtain to the goals of comfort and energy saving by considering the real characteristics of air conditioning system. Therefore, the FCM control method could be considered as an efficient strategy to multiple-input and multiple-output (MIMO) and nonlinear system with strong cross coupling effect between the parameters of the system. It is observe from simulation results that thermal comfort achieved and energy efficiency obtained by eliminating the humidifier in tropical countries.
\end{abstract}

Keyword: Direct expansion air conditioning (DX A/C); MIMO; Fuzzy cognitive map (FCM); Nonlinear; Generalized predictive model (GPC) 